\title{
The Evolution and Design of Flat-Panel Loudspeakers for Audio Reproduction
}

\author{
MiChAEL C. HEILEMANN, ${ }^{1}$ AES Associate Member, DAVID A. ANDERSON, ${ }^{2}$ AES Member, \\ (mheilema@ur.rochester.edu) \\ (danderson@ara.com) \\ STEPHEN ROESSNER, ${ }^{1}$ AES Member AND MARK F. BOCKO, ${ }^{1}$ AES Member \\ (stephen.roessner@rochester.edu) (mark.bocko@ rochester.edu) \\ ${ }^{1}$ University of Rochester, Rochester, NY, USA \\ ${ }^{2}$ Applied Research Associates, Inc., Littleton, CO, USA
}

\begin{abstract}
The underlying physics and the design of loudspeakers that radiate sound through the bending vibrations of elastic panels, here referred to generically as flat-panel loudspeakers, are reviewed in this paper. The form factor, reduced weight, and aesthetic appeal of flat-panel speakers have made them a topic of interest for more than 90 years, but these advantages have been overshadowed by acoustical shortcomings, specifically the uneven frequency response and directivity in comparison to conventional cone-radiator loudspeakers. Fundamentally, the design challenges of flat-panel speakers arise from the intrinsically large number of mechanical degrees of freedom of a panel radiator. A number of methods have been explored to compensate for the acoustical shortcomings of flat-panel speakers, such as employing inverse filters, equalization, canceling mechanical resonances with actuator arrays, and modifying the panel material, shape, structure, and boundary conditions. Such methods have been used in various combinations to achieve significant audio performance improvements, and carefully designed flat-panel loudspeakers have been rated in blind listening tests as competitive with some prosumer-grade conventional loudspeakers. This review presents a brief historical account of the evolution of flat-panel loudspeakers and summarizes the essential physics and design methodologies that have been developed to optimize their fidelity and directional response.
\end{abstract}

\section{O INTRODUCTION}

High-fidelity loudspeakers designed to accurately reproduce the temporal and spectral characteristics of recorded audio signals typically exhibit smooth frequency response characteristics in both on-axis and off-axis sound pressure measurements $[1,2]$. The common voice-coil, coneradiator loudspeaker has proven to be a highly effective means to achieve the transient and frequency response characteristics required for high-fidelity sound reproduction since its introduction in the early 20th century [3]. The one-dimensional pistonic motion of the cone uniformly displaces air, which simplifies the tuning process. However, to achieve smooth frequency response both on-axis and offaxis, conventional cone-radiator loudspeakers are designed with multiple drivers to avoid beaming at high frequencies when the wavelength of the sound waves is comparable to the diameter of the cone. Properly tuned, ported enclosures are often employed to enhance low-frequency response [4].
Shortly after the introduction of the cone-radiator loudspeaker, researchers began investigating alternative electroacoustic transducer designs to address one or more of their perceived shortcomings, in particular their size and weight. The idea of the first flat-panel loudspeaker was born out of these investigations [5] and has since evolved to include products used in the fields of home theater, public address systems, and telecommunications. The basic concept is simple - to radiate sound by exciting the bending vibrations of a panel using one or more small force actuators affixed to the panel surface. However, in comparison to cone-radiator loudspeakers, flat-panel loudspeakers have not achieved widespread commercial acceptance due to deficiencies in their sound quality $[6,7]$.

In contrast to a cone-radiator loudspeaker, which is designed to have a single mechanical degree of freedom within its intended frequency range, a typical flat-panel speaker has many degrees of freedom in the audio frequency band (20 Hz to $20 \mathrm{kHz}$ ), perhaps tens of thousands depending 
upon the panel dimensions and materials. While this may seem like an impossibly high number of degrees of freedom to control, external factors such as the panel's internal friction and energy losses at supports reduce the number of isolated resonant modes at high frequencies. However, failure to control the low-frequency isolated bending modes can lead to irregular on-axis and off-axis frequency responses.

Throughout the history of their development, the sound quality deficiencies of flat-panel loudspeakers have outweighed their form factor advantages, and consumer products were designed to accommodate the space required to integrate high-quality cone-radiator loudspeakers. However, advances in display technology are making devices thinner, lighter, and more portable, so interest in low-profile loudspeakers has been rekindled. Specifically, devices have begun to appear in which flat-panel loudspeaker technology has been employed to make displays perform double duty as loudspeakers. Companies such as LG and Sony have introduced mobile phones [8, 9] and televisions [10-13] that employ the bending motion of the display itself to generate sound.

This paper provides a brief historical review of the evolution of flat-panel loudspeakers and an assessment of the current state of the technology for applications in modern electronic devices. We have made efforts to be reasonably inclusive of the historical development of the field; however, there is an extensive patent literature in this field, and we apologize in advance if we have overlooked anyone's contributions. Our main intentions are to provide a tutorial on the vibroacoustics of flat-panel loudspeakers, to outline their intrinsic shortcomings and challenges, and to provide a framework for finding design solutions to these problems. We hope that this will prove useful to the community of loudspeaker designers tackling the challenges of integrating sound into a range of modern devices. We begin with a review on the vibroacoustics of point-driven panels to outline the fundamental challenges involved in designing flat-panel loudspeakers and to provide a mathematical foundation for some of the solutions presented in later sections.

\section{VIBROACOUSTICS OF PANELS}

Following [14] and [15], the equation of bending motion for a thin, damped, isotropic panel may be expressed as

$$
\begin{aligned}
D \nabla^{4} u(x, y, t)+\rho h \frac{\partial^{2} u(x, y, t)}{\partial t^{2}}+ & b \frac{\partial u(x, y, t)}{\partial t} \\
& =P(x, y, t) .
\end{aligned}
$$

where $u(x, y, t)$ is the out-of-plane displacement of the the panel at location $(x, y)$ and time $t, h$ is the panel thickness, $P$ is the external pressure applied to the panel, $\rho$ is the density of the panel, $b$ is the damping coefficient, and $D$ is the bending stiffness per unit width given by

$$
D=\frac{E h^{3}}{12\left(1-v^{2}\right)},
$$

with Young's modulus $E$ and Poisson's ratio $v$. The externally applied pressure on a flat-panel loudspeaker generally

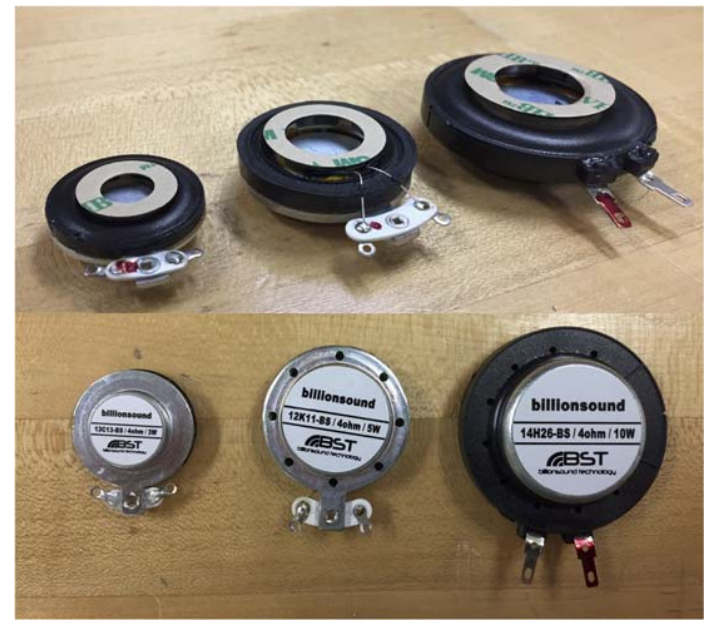

Fig. 1. Front and rear views of 3-W, 5-W, and 10-W moving-coil actuators produced by Billionsound [16]. Reproduced from [22].

is provided by one or more moving-coil actuators, such as those shown in Fig. 1, manufactured by Billionsound [16], although piezoelectric actuators also may be employed to excite the bending vibrations of panels and have been used in applications such as active vibration control [17-21]. A moving-coil actuator driven by a sinusoidal signal at frequency $\omega$ may be approximated as a point force $\tilde{F} e^{j \omega t}$ when the wavelength of the bending wave excited in the panel is large compared to the diameter of the actuator.

Once the boundary conditions have been specified, the solutions of Eq. (1) may be presented in many forms. However, the challenges of designing flat-panel speakers are made most apparent by expressing the panel response as a superposition of resonant modes. Following Fuller [23], the response of the panel $\tilde{u}_{i}(x, y, \omega)$ due to excitation by the $i^{\text {th }}$ actuator located at position $\left(x_{i}, y_{i}\right)$ is given by

$$
\tilde{u}_{i}(x, y, \omega)=\sum_{r=1}^{\infty} \tilde{F} \tilde{\alpha}_{i r} \Phi_{r}(x, y),
$$

where $\Phi_{r}(x, y)$ is the spatial response of each resonant mode $r$ and $\tilde{\alpha}_{i r}$ is the frequency dependent amplitude of each mode due to excitation by the $i^{\text {th }}$ actuator. The spatial response of each mode is determined by the boundary conditions of the panel. For example, the mode shapes of the resonant modes for a panel with simply supported edges are products of sinusoidal functions in the vertical and horizontal dimensions. From [15], the amplitude of each mode may be expressed in terms of the actuator location, the surface area $S$ of the panel, the resonant frequency $\omega_{r}$ of the mode, and the quality factor $Q_{r}$ of the mode as

$$
\tilde{\alpha}_{i r}=\frac{4 \Phi_{r}\left(x_{i}, y_{i}\right)}{\rho h S\left(\omega_{r}^{2}-\omega^{2}+j \omega_{r} \omega / Q_{r}\right)} .
$$

The moving-coil actuators shown in Fig. 1 act as masses supported by spring-like suspensions, which give the actuators their own natural resonant frequencies. When affixed to a panel, these actuator resonances couple to the resonances of each panel mode, causing the resonances of the coupled system to occur at different frequencies than those 


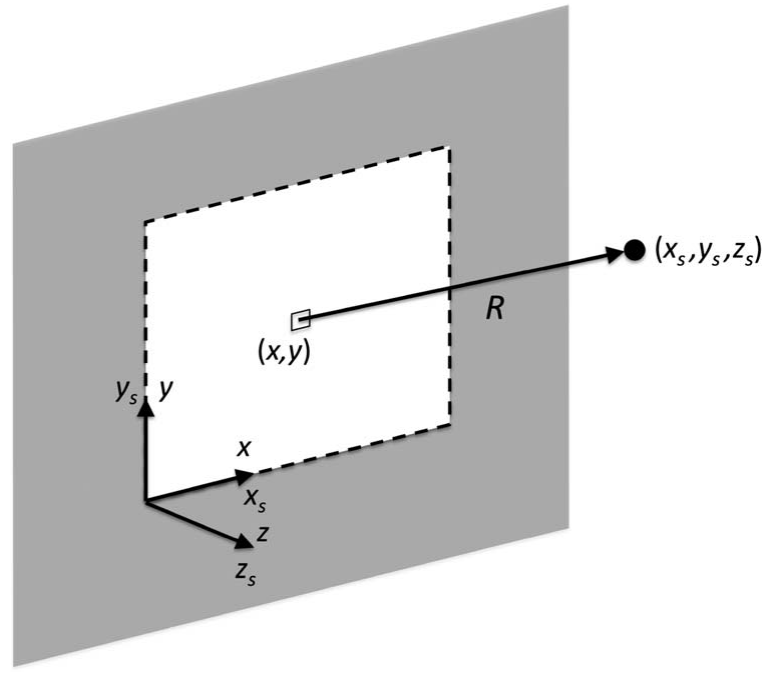

Fig. 2. Coordinate layout of a panel with an infinite baffle and a point in space used for the Rayleigh integral. Reproduced from [22].

of the uncoupled systems. This effect was modeled in [24] and can be reduced by selecting actuators whose individual resonances are below that of the first panel bending mode.

The total response $\tilde{u}(x, y, \omega)$ of a panel excited by an array of $N$ actuators is given by the superposition of the responses to each actuator individually as

$$
\tilde{u}(x, y, \omega)=\sum_{i=1}^{N} \tilde{u}_{i}(x, y, \omega)=\sum_{i=1}^{N} \sum_{r=1}^{\infty} \tilde{F} \tilde{\alpha}_{i r} \Phi_{r}(x, y) .
$$

\subsection{Sound Radiated by Panels}

The mechanical response of a panel $u(x, y, t)$ excited at frequency $\omega$ is related to the acoustical response of a panel via the Rayleigh integral [25], which gives the sound pressure $p\left(x_{s}, y_{s}, z_{s}, t\right)$ at a location $\left(x_{s}, y_{s}, z_{s}\right)$ in space as

$$
\begin{aligned}
& p\left(x_{s}, y_{s}, z_{s}, t\right)=\frac{-\omega^{2} \rho_{0}}{2 \pi} \iint_{S} \frac{u(x, y, t-R / c)}{R} d s, \\
& R=\sqrt{\left(x_{s}-x\right)^{2}+\left(y_{s}-y\right)^{2}+z_{s}^{2}},
\end{aligned}
$$

where $\rho_{0}$ is the density of air, $c$ is the speed of sound in air, and $R$ is the distance from a point on the panel $(x, y)$ to a point in space $\left(x_{s}, y_{s}, z_{s}\right)$, as shown in Fig. 2. The Rayleigh integral divides the surface area $S$ of the panel into subregions, each with area $d s$. Each subregion acts as an elementary point-source acoustic radiator, and the total acoustic response at a point $\left(x_{s}, y_{s}, z_{s}\right)$ in space is computed by summing the acoustic contributions of each point-source radiator at the selected point in space. For this calculation, it is assumed that the panel is placed in an infinite baffle to decouple the sound fields radiated from the front and rear of the panel. Removal of the baffle can result in a decreased radiation efficiency in the far field at frequencies for which the wavelength of the radiated sound is longer than the bending wavelength on the panel [26].

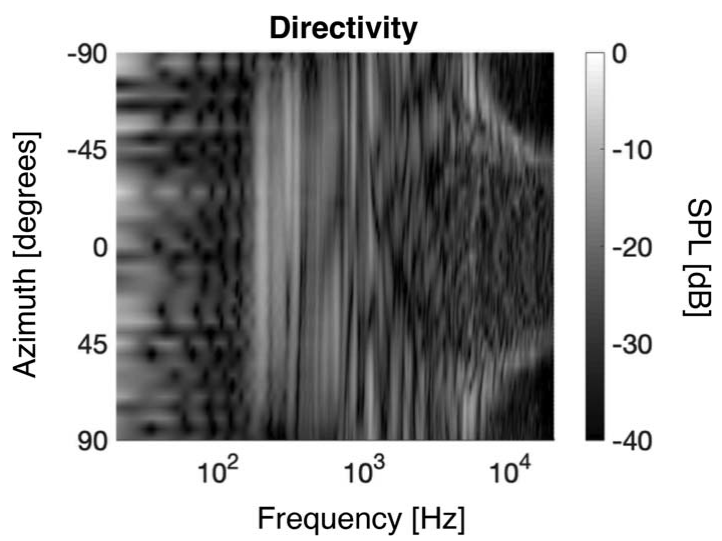

Fig. 3. Plot of the radiated sound pressure from an acrylic flatpanel radiator as a function of frequency and angle measured $1 \mathrm{~m}$ from the panel in the median plane. Note that zero degrees is directly in front of the panel. Reproduced from [22].

The physical behavior of panel speakers has been described in various papers [27-29], and the resulting acoustic radiation has been studied in depth [30-35]. Numerical simulations of Eq. (6) show that panel speakers typically produce an irregular radiated sound field that is highly depended on frequency and the position of the listener relative to the loudspeaker. This is shown in Fig. 3, where a polar view of the normalized sound pressure level (SPL) at radius of $1 \mathrm{~m}$ from the center of the panel is shown as a function of angle and frequency for a $38 \mathrm{~cm} \times 28 \mathrm{~cm} \times 0: 6 \mathrm{~cm}$ acrylic panel excited by a single actuator.

In addition to the artifacts of a flat-panel loudspeaker's modal response, the sound radiation properties of a panel are strongly dependent on the coincidence frequency of the panel $[25,14,23,27]$. In air, at a constant temperature, sound propagates with a frequency-independent wave speed $c$. However, panels are dispersive, and the speed of a bending wave propagating in a panel $c_{\text {panel }}$ is given by

$$
c_{\text {panel }}=\left(\frac{D \omega^{2}}{\rho h}\right)^{1 / 4}=\left(\frac{E h^{2} \omega^{2}}{12 \rho\left(1-\nu^{2}\right)}\right)^{1 / 4},
$$

which comes from Eq. (1). From Eq. (8), it is apparent that the bending wave speed in a panel is a function of the panel material, thickness, and frequency. The dispersive nature of panel-bending waves implies the existence of a coincidence frequency $\hat{f}_{\mathrm{c}}$, in which the wave speed on the panel is equal to the wave speed in air. Following [36], the coincidence frequency is given by

$$
\hat{f}_{\mathrm{c}}=\frac{c^{2}}{2 \pi} \sqrt{\frac{\rho h}{D}}=\frac{c^{2}}{2 \pi} \sqrt{\frac{12 \rho\left(1-v^{2}\right)}{E h^{2}}} .
$$

Below the coincidence frequency, the radiation response of the panel is determined by the radiation patterns associated with the various panel bending modes. This can be seen in Fig. 4 at frequencies between approximately 170 $\mathrm{Hz}$ (the fundamental resonance of the panel) and $6,000 \mathrm{~Hz}$, where there are lobes of strong radiation across the azimuth corresponding to the various bending modes. Above coincidence, a plane wave is radiated from the panel surface. 

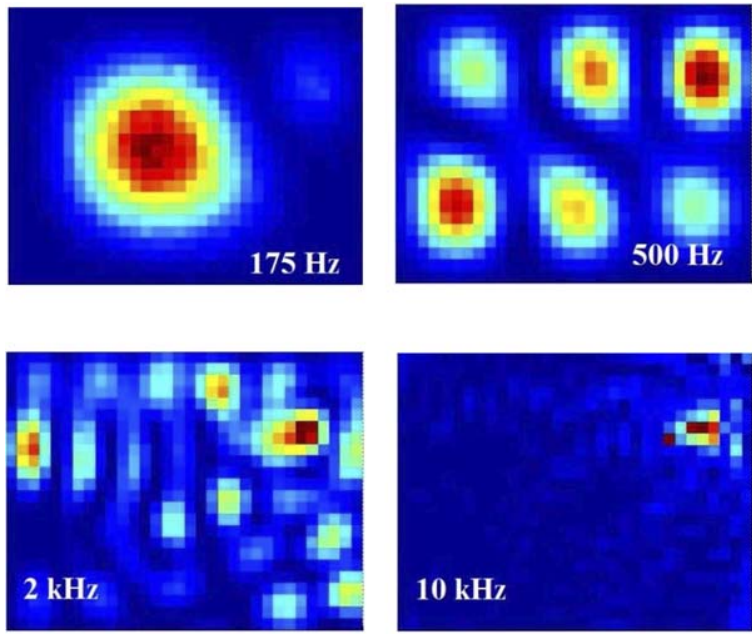

Fig. 4. Laser vibrometer measurements of a $38 \mathrm{~cm} \times 28 \mathrm{~cm} \times$ $0.6 \mathrm{~cm}$ acrylic panel with fixed edges. Bright spots with dark circles in the center indicate regions of high displacement, while the dark regions surrounding the bright spots indicate regions of low displacement. The vibrations begin to localize around the driving element in the upper right corner above an excitation frequency of $2 \mathrm{kHz}$. Reproduced from [37] with the permission of the Acoustical Society of America.

Since the bending wavelength is longer than the wavelength of sound in air above coincidence, the radiated plane wave is emitted at an angle $\vartheta$ with respect to the normal given by

$$
\vartheta=\sin ^{-1}\left(\frac{c}{c_{\text {panel }}}\right)
$$

An equivalent expression may be found in [27] and [15] using wavelength and wavenumber parameters, respectively. At coincidence, the wavelengths and propagation speeds are the same for both air and the bending wave; therefore, strong radiation appears at $+90^{\circ}$ and $-90^{\circ}$ as shown beginning at approximately $6,000 \mathrm{~Hz}$ in Fig. 3. As the excitation frequency increases, the angle of the radiated plane wave tends toward $0^{\circ}$.

\subsection{Modal Overlap}

From Eqs. (5) and (6), the acoustic response of a panel can be computed as a sum of the responses of individual modes. In two and three-dimensional distributed systems such as panels and rooms, the frequency separation between modal resonances decreases with increasing resonant frequency. This means that at low frequencies, the mechanical frequency response of a panel clearly shows the contributions of isolated individual modes, while at high frequencies, the response of the panel is characterized by the superposition of many overlapping modes, i.e., when the mode separation is much less than the frequency bandwidth of the individual modes. In this regime, the vibrations of the panel remain localized around the driving element [37], and the modal response is less apparent. The simultaneously excited modes form a band-limited Fourier series reconstruction of a two-dimensional delta function on the panel at the location of the driving element.

This is shown in Fig. 4, where a laser vibrometer was used to measure the mechanical response at various frequencies of a $38 \mathrm{~cm} \times 28 \mathrm{~cm} \times 0.6 \mathrm{~cm}$ acrylic panel with fixed edges and an actuator placed in the upper right corner of the panel. The effect is similar to the transition in room response from modal response at low frequencies to the higher-frequency region, in which there are many overlapping room modes and a statistical description of the room response is more applicable [38].

The transition frequency between the low-frequency modal region and the high-frequency localized response is determined by a quantity referred to as modal overlap (MO) [39], which is a measure of the number of modes excited at any given frequency. A mode with index $r$ is considered to be excited at frequency $\omega$ if $\omega_{r-}<\omega<$ $\omega_{r+}$, where $\omega_{r-}$ and $\omega_{r+}$ are the lower and upper $-3 \mathrm{~dB}$ points of the resonant peak associated with mode $r$. The transition frequency is defined as the frequency at which the MO is always greater than or equal to 1 . In other words, there are always at least two simultaneously excited modes above the transition frequency. The MO (and thus the transition frequency between regions) is highly dependent on the boundary conditions, surface area, thickness, and amount of damping in the panel. In general, large, thin panels with high damping and free edges will have the lowest transition frequencies.

Knowing the MO transition frequency is critical in the design of flat-panel loudspeakers. The next several sections will outline a number of mechanical and electrical designs to improve the performance of flat-panel loudspeakers. Each of the methods discussed are useful under certain conditions and assumptions. In practical applications, a combination of methods is needed in different frequency bands defined by the $\mathrm{MO}$ factor.

\section{IMPROVEMENTS THROUGH MECHANICAL DESIGN}

Early published work on sound generation by the bending vibrations of panels begins with patents by Lane [40, 41], Nevin [42], and Kroesen [43]. The novelty of creating sound from preexisting structures such as movie theater screens or artwork displayed in the home is evident. Unfortunately, the physics of such loudspeakers was not appreciated fully, and as a consequence, the sound quality likely was deficient, judging from the ephemeral nature of the proposed designs. Rectangular panels with constrained edges display sharp peaks in their frequency response at the resonant mode frequencies. The presence of such resonances, which are apparent below the transition frequency, is the source of the "hollow" sound often attributed to flatpanel loudspeakers. This implies that designing panels with the transition frequency as low as possible, either by increasing the density of modes through mechanical design or increasing mechanical damping, is a promising direction to pursue. However, both methods have their limitations, and in practice, it proves difficult to construct panels that 


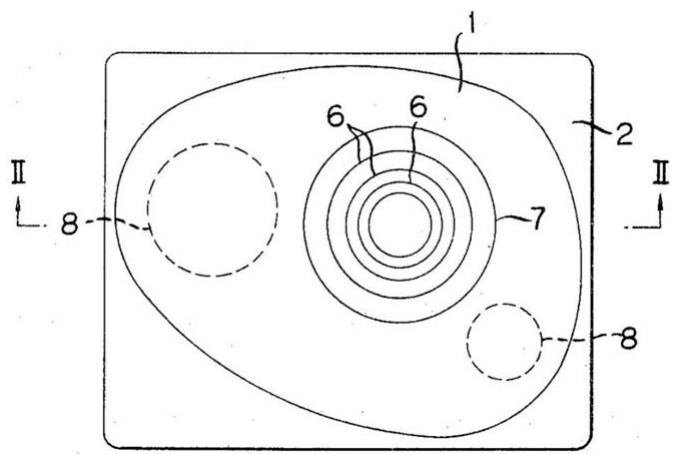

Fig. 5. A diagram of the flat-panel loudspeaker patented by Sotome. Reproduced from [44].

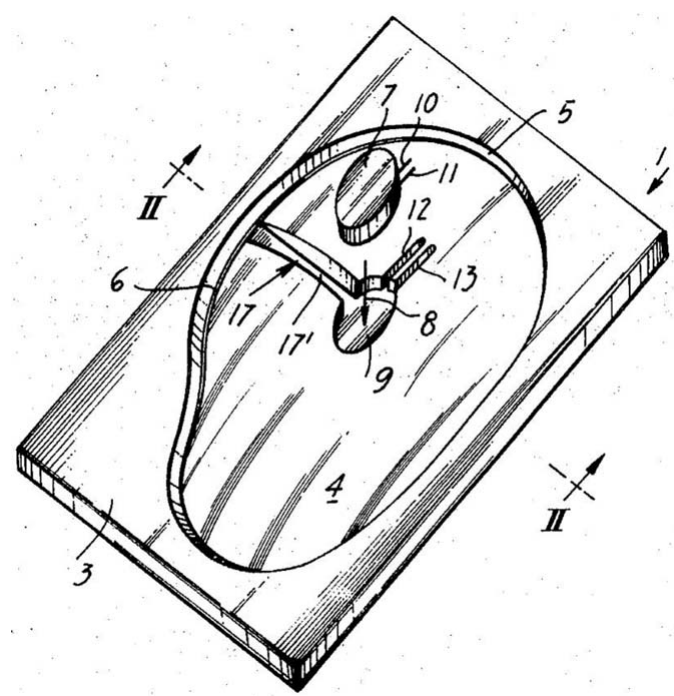

Fig. 6. A diagram of the loudspeaker patented by Bertagni. Reproduced from [47].

do not display isolated modal behavior within the audible frequency band.

In the 1970s, a good deal of work was published related to enhancing flat-panel loudspeaker design by mechanical tuning methods. Yamaha's "Natural Sound" loudspeaker was made from a panel whose irregular shape, shown in Fig. 5, was designed to control the frequencies and density of modes to create more pleasing sonic characteristics, as described in the patents of Sotome [44] and Mochida [45].

Bertagni Electroacoustic Systems, led by Jose Bertagni, was prolific in researching and commercializing tunedpanel loudspeakers. His patents describe panels with improved mechanical aspects, such as increased stiffness at the excitation point to increase high-frequency fidelity [46], a variable-thickness panel for modifying the propagation of waves in the structure [47], multiple connected vibrating plates [48], a modified panel structure [49], and supports or mounting methods designed to enhance the frequency response [50]. Fig. 6 shows a modified panel structure from [47].

A patent by Heron [51] involving sound radiation from a lightweight aluminum-honeycomb panel led to the for- mation of the company New Transducers Limited (NXT), whose name is closely associated with flat-panel loudspeakers referred to as Distributed-Mode Loudspeakers (DMLs). It is common in many audio circles to hear the terms "flatpanel loudspeaker" and "DML" used interchangeably. In this context, we use the term DML to describe a specific flatpanel loudspeaker design that attempts to generate modes that are evenly distributed spatially on the panel and in the frequency domain as described in [31]. NXT's patents concerned the optimal material density for panels [52], as well as optimal shapes and exciter mounting positions for various applications [53]. The idea of designing panels in which the motion was uniform (or pistonic) at low frequencies but bending mode at high frequencies appeared in work by Harris [54] and Beer [55].

Additionally, Hill patented a panel speaker with different material densities in different areas [56], and a speaker using an anisotropic diaphragm was patented by Lock et al. [57]. Research has also concerned optimization of a panel's aspect ratio to achieve maximum coupling between exciters and the spectrum of panel modes [58] and attaching masses to a panel in an optimal way to achieve a flat frequency response [59].

A problem common to these loudspeaker designs remains the modal response of the panel at low frequencies within the audio range. Such modal resonances remain the root cause of the hollow sound often criticized in flat-panel speakers. A marked advantage of some of the proposed DML designs was to employ highly compliant panels with free edges, as specified in Heron's patent [28], which moves the modal crossover frequency to below $20 \mathrm{~Hz}$, the lower limit of the audio band, thereby eliminating the objectionable audio artifacts arising from modal response. The MO crossover point of a smaller panel whose edges are fixed or hinged likely will be within the audio frequency band, leading to audible artifacts and thereby motivating the mechanical tuning methods described above.

\section{IMPROVEMENTS THROUGH SIGNAL PROCESSING}

While the majority of mechanical improvements focus on extending the effective frequency range, improving efficiency, and tuning of the overall frequency response, the focus of electronic or signal-processing intervention is typically an attempt to flatten the frequency response or improve the spatial coverage of the loudspeaker. Unfortunately, if the acoustic radiation from a flat-panel loudspeaker is sampled at a single point in space, changing the observation point may show a much different frequency response characteristic [32]. Since the acoustic response of flat-panel loudspeakers may exhibit great variability depending on the measurement point, papers that report the application of inverse filters derived from impulse response measurements taken at a given point in space may prove to be ineffective when the listener moves to a different point relative to the loudspeaker [60-62].

A possible electronic intervention that allows the designer greater spatial control is to use multiple strategically 

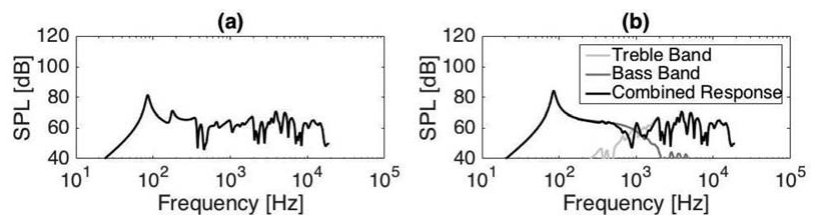

Fig. 7. On-axis acoustic frequency response simulations for a panel loudspeaker excited by (a) a single force actuator and (b) the modal crossover technique described in [64]. Reproduced from [22].

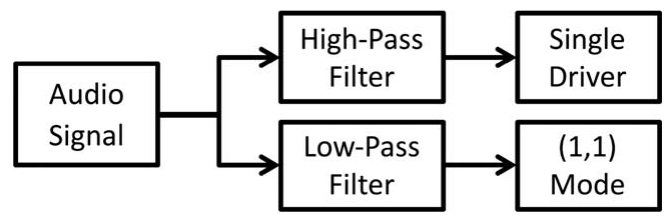

Fig. 8. Signal flow of a flat-panel loudspeaker employing a modal crossover network.

placed exciters to excite the panel. Zhang et al. [63] demonstrated that an improved single-point frequency response was possible by optimizing the locations and sizes of eight exciters on a multiexciter DML. Multiexciter configurations and signal processing also may be combined to drive vibration patterns with desirable sound radiation properties. A method for physically tuning the frequency response of a panel using an array of exciters to enhance or attenuate certain bending modes of the panel was described in [64]. It later was demonstrated that an array with optimized exciter locations [65] achieved a more uniform radiation pattern in front of a panel at low frequencies [66].

A simulation comparison between the on-axis acoustic frequency response of a panel loudspeaker excited by a single driver and a panel loudspeaker employing the arraydriven method described in [64] is shown in Fig. 7. A crossover network was used for the array-driven panel so that the low-frequency band was reproduced only through the lowest bending mode of the panel, while the highfrequency band was reproduced through a single actuator. A diagram of the signal flow for the loudspeaker is shown in Fig. 8. In this simulation, the crossover frequency between the bands was set at $1.1 \mathrm{kHz}$. The resonant peaks located between $100 \mathrm{~Hz}$ and $1 \mathrm{kHz}$ are eliminated in the combined response of the loudspeaker employing the actuator array.

For a loudspeaker in which tuning control is important, such as the array-driven speaker described above, the required number of exciters has been shown to be proportional to the MO crossover point [37] since, above this frequency, vibrations on a panel are essentially nonmodal.

\section{PERCEPTUAL EVALUATIONS}

Laboratory measurements generally can be conducted more quickly and easily than blind human listening evaluations. Therefore, the identification of objective measurements that demonstrate strong correlations with loud- speaker listener preference has been of interest to many researchers.

The first research into loudspeaker listener preference was performed by F. H. Brittain [67-69], who identified key objective measurements that influence listener preference, such as frequency response, harmonic distortion, transient distortion, and intermodulation distortion. The relative importance of these measurements as indicators of loudspeaker quality has been the subject of much debate.

In the 1980s, Toole demonstrated that inconsistencies in listening tests were the root cause for supporting one technical evaluation scheme over another [70]. He demonstrated that variability in subjective evaluations can stem from environmental factors, such as the loudspeaker position, listener position, and type of room; psychological factors, such as the subject's hearing ability, knowledge of the products, and prior experience with the test; or flaws in the experimental procedure, such as experimenter bias, subject memory, and context effects [71]. He also showed that listening tests can produce reliable subjective data by eliminating as many of these variables as possible through double-blind tests in a consistent environment [72]. Comparing the objective data with the controlled subjective data allowed the most important objective measures to be realized.

For example, amplitude response measurements with one-third octave smoothing were able to identify low-rated loudspeakers but lacked the resolution necessary to identify the highest-rated loudspeakers. Metrics such as total sound power also were unable to separate the highest-quality loudspeakers [73]. Independent evaluations of the smoothness and flatness of on/off-axis amplitude response measures with at least one-tenth octave resolution demonstrated the highest predictive properties. Building on Toole's papers, researchers at Harman International [1, 2] created a multiple regression model to predict listener preference ratings of loudspeakers from a set of anechoic measurements. Their generalized model showed a high correlation $(\mathrm{r}=0.86)$ with subjective preference ratings.

Unlike traditional loudspeakers, there have been relatively few studies on the subjective qualities of flat-panel loudspeakers. One perceptual test by Flanagan and Taylor [74] found empirically that the ratio of direct to reflected sound is a function of the type of loudspeaker, not just the distance from the listener to the speaker. Similarly, Flanagan and Harris [75] showed that there is a loudness perception difference between a flat-panel speaker and a traditional speaker, both objectively and subjectively. Both of these tests were performed in an untreated rectangular room, approximately $4 \mathrm{~m}$ by $6 \mathrm{~m}$, with a reverberation time (RT60) of $0.463 \mathrm{~s}$ at $1 \mathrm{kHz}$. They argue that DMLs need an increased amplitude compared with traditional loudspeakers due to the diffuse nature of wall reflections to provide a more accurate distance perception. Harris and Flanagan [76, 77] also demonstrated that DMLs lessen the detrimental effects of the room reflections on stereo localization.

Recently, the evaluation model developed in $[1,2]$ was applied to a traditional coaxial loudspeaker, a commercial panel loudspeaker, and a panel loudspeaker that 

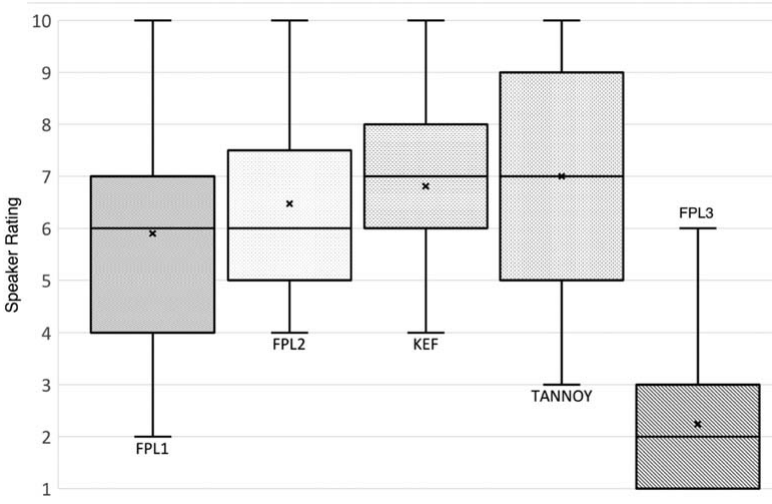

Fig. 9. Preference ratings of damped panels with modal crossover networks (FPL1 and FPL2), a commercial flat-panel speaker (FPL3), a Kef LS50, and a Tannoy PBM 6.5 II determined by 21 subjects in a blind listening test. Reproduced from [79].

could switch between single-actuator excitation and array/multiactuator excitation with a modal crossover network [78]. Though the panel employing the modal crossover network greatly outperformed the single-actuator panel and the commercial panel, it fell well short of the predicted preference rating for the traditional coaxial loudspeaker due to its uneven high-frequency response. To smooth the high-frequency response, damping was added to panels with modal crossover networks (FPL1 and FPL2) by adhering constrained viscoelastic material to the panel surface.

A set of 21 listeners compared these panels to a Kef LS50 coaxial two-way bookshelf speaker, Tannoy PBM 6.5 II conventional two-way book shelf speaker, and a commercial flat-panel loudspeaker (FPL3) in a blind listening test. The ratings on a 1-10 scale are shown in Fig. 9, with the modal crossover panels rating favorably compared to the conventional speakers and greatly outperforming the commercial flat-panel speaker [79]. The maximum and minimum ratings are indicated by the whiskers of each box, the median rating is given by the solid line in the box, and the average rating for each speaker is indicated by an " $x$ ". The box represents the middle $50 \%$ of the ratings for each speaker.

While these initial results are promising, a great deal of work remains in this area. There are a number of other mechanical and electrical improvements mentioned in the previous sections that may be evaluated perceptually. It is unclear how the larger surface area of flat-panel speakers compared to conventional speakers affects listener preference. In addition, prediction models such as [1,2] may need to be adapted to account for Doppler distortions, which may arise because a single panel is used as a broadband radiator. This may cause the high-frequency signals to be modulated by the low-frequency excursions of the panel.

\section{SPATIAL AUDIO}

Increasingly, spatial audio applications have employed flat-panel loudspeakers. The large, distributed surface and

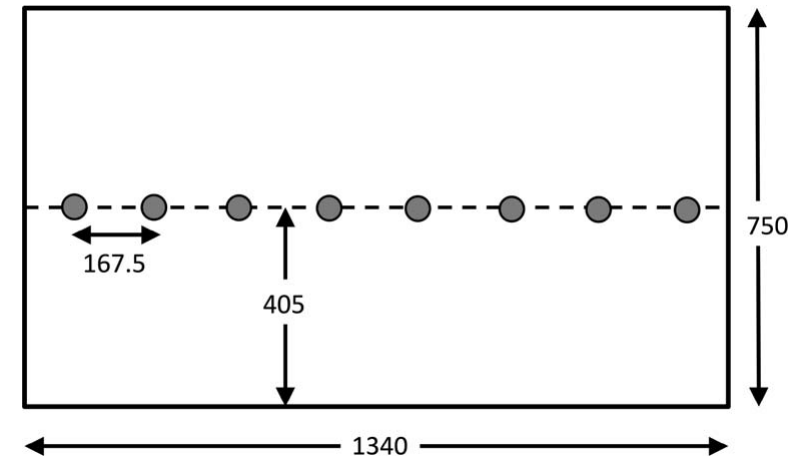

Fig. 10. Dimensions (mm) and actuator spacing for a foam board multiactuator panel (MAP). Reproduced from [61].

low cost of flat-panel speakers would seem, at least superficially, to have advantages over multispeaker arrays conventionally employed for 3D sound-rendering schemes such as wave field synthesis (WFS) [80]. The flat-panel methods considered employed multiple force exciters mounted on a large panel, with each exciter individually driven by an appropriate signal.

\subsection{Multiactuator Panels}

In the early 2000s, researchers began exploring the use of DML panels as platforms for WFS. One of the first publications on this subject was by van Rooijen [81], where the advantages and disadvantages of using DML panels for WFS were discussed. Among the advantages listed were the light weight, easy construction, potential for architectural integration, and the ability to extend the frequency response while avoiding spatial aliasing by employing highly damped panels with an array of closely spaced force exciters. The disadvantages listed were well-known critiques of DML panels, including weak bass response and modal resonance distortion of the impulse response.

Boone [61] coined the phrase "multiactuator panels" (MAPs) to describe multiexciter DML panels. He explored the WFS potential of panel loudspeakers using arrays of small, single-exciter DML panels and larger, highly damped MAPs made of foam board. He determined that, although both MAPs and arrays of DMLs could be used for WFS reproduction, MAPs were advantageous because the large surface area gave improved bass response. An example of the dimensions and actuator spacing for the MAPs used in [61] is shown in Fig. 10.

A number of techniques have been employed to equalize the response of MAPs in an effort to overcome the irregular frequency response and resonant impulse response inherent in DML panels. These include inverse and infinite impulse response filtering developed by Corteel et al. [82] and Pueo et al. $[83,84]$ and active vibration control techniques developed by Ho and Berkhoff [85]. Pueo et al. [86] used the psychoacoustic principle of the missing fundamental to improve the bass response of MAPs so that listeners could perceive frequencies an octave below the natural MAP low cut-off frequency. Though successfully implemented, many 

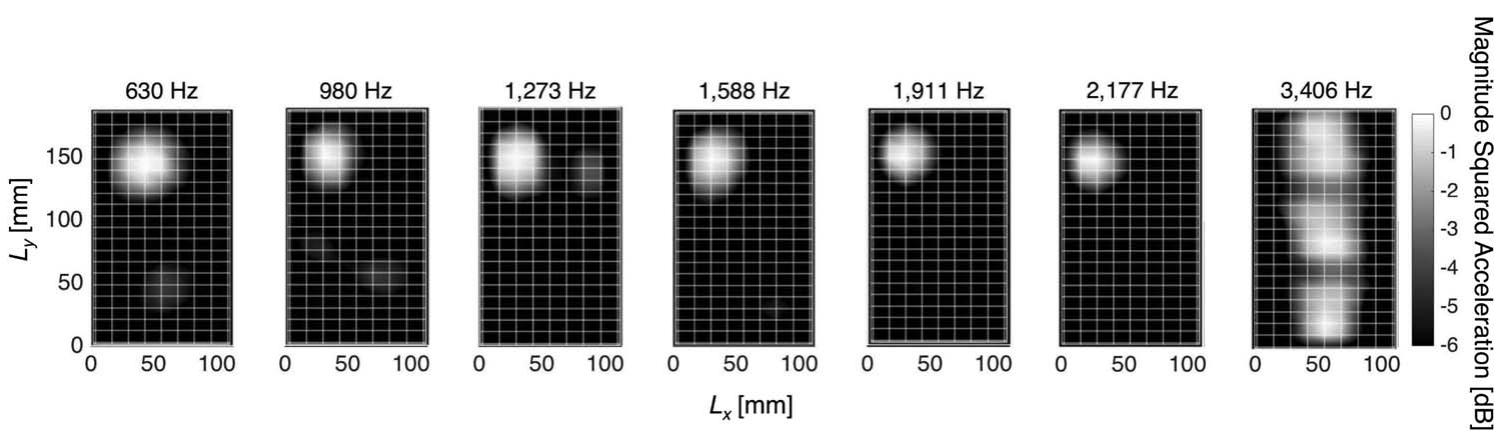

Fig. 11. Laser vibrometer scans of a vibration localized to the upper-left corner of an aluminum panel with fixed edges. The panel response in each plot is presented in $\mathrm{dB}$ relative to the maximum acceleration energy of the panel at the frequency shown. Reproduced from [94].

of these techniques are limited in that they are only effective at a single point in listening space.

The structural vibration characteristics of MAPs were observed by Kuster et al. [87], who used laser vibrometer impulse response measurements to show that the vibrations of an actuator are relatively unaffected by neighboring exciters and the panel edges. In addition, analysis of the vibrometer scans showed that the panel vibrations and the resulting acoustic emanations were confined to areas surrounding the actuators, leading the authors to conclude that the size and damping of MAPs were such that individual exciters could be treated as independent sources for WFS purposes. It was noted, however, that force actuators located at different positions around the panel may produce different radiation patterns, which could lead to phasing issues between the sources.

Further work on the inverse filter method was performed by van Dorp Schuitman and de Vries [88], who developed a faster algorithm based on singular value decomposition; however, this method was limited to correcting the acoustic response at a single point in space. Recently, Rébillat et al. [89] investigated the use of large MAPs for virtual reality displays and projector screens. These panels were treated as infinite plates (high MO), and thus, vibrations were shown to be localized around the excitation point. Each exciter was treated as a monopole source, and it was concluded that such panels are able to employ WFS to create virtual acoustic scenes. Jeon et al. [90] used the localization factor described in [37] to optimally place exciters on MAPs for beamforming. A review of MAPs for WFS was published in 2010 [91].

\subsection{Localized Source Rendering}

Spatial audio also may be achieved by rendering primary sources directly at their desired locations on the panel. In the high MO region, this task is simple, since the vibrations associated with a particular actuator remain localized to the immediate region around the actuator. In regions of low $\mathrm{MO}$, localizing vibrations requires prior knowledge of the panel's vibration characteristics and an array of properly tuned actuators to force the desired panel response at a particular excitation frequency.
The bending modes of a panel form an orthogonal basis for representing the spatial response of the panel. This implies that any localized vibration region may be reconstructed by selectively exciting a weighted superposition of the panel's bending modes. A similar idea was used to localize haptic vibrations on panels with a large array of actuators distributed along the perimeter of the panel [92]. From [93], an array of $N$ actuators can independently excite $N$ modes, which can serve to create a band-limited Fourier series representation of any given panel vibration profile. Spatial aliasing known as "spillover" [23] can occur when modes outside of the prescribed set are excited.

If the spatial response of each mode $\Phi_{r}(x, y)$ is known, the modal amplitudes $\alpha_{r}$ needed to reconstruct a target spatial response $\varphi(x, y)$ may be determined by a Fourier series expansion,

$$
\alpha_{r}=\frac{4}{S} \iint_{S} \varphi(x, y) \Phi_{r}(x, y) d s
$$

A simple second-order filter may be designed to govern the magnitude and phase behavior of each actuator based on the actuator's position on the panel, the target shape to be rendered, and the material properties of the panel. These filters will maintain a spatially band-limited representation of the target vibration region at all frequencies within the controllable frequency bandwidth of the actuator array [94]. The controllable bandwidth is simply the band of frequencies spanned by the set of panel modes that are being controlled by the actuator array. The primary acoustic source rendered by the localized vibrating region may be equalized and dynamically moved to other spatial locations on the panel $[95,96]$. An example of a source localized by eight actuators in the upperleft corner of a 1-mm-thick aluminum panel of dimensions $L_{x}=113 \mathrm{~mm}$ and $L_{y}=189 \mathrm{~mm}$ with fixed edges is shown in Fig. 11. The target vibration profile is maintained until the frequency of the first spillover mode $(1,6)$ at $3,406 \mathrm{~Hz}$.

The method described above is limited in that the filters for each actuator are designed using an analytical model for the spatial response of each mode. This requires careful construction of the panel so that the modes match those predicted by the model. The system can be made more 


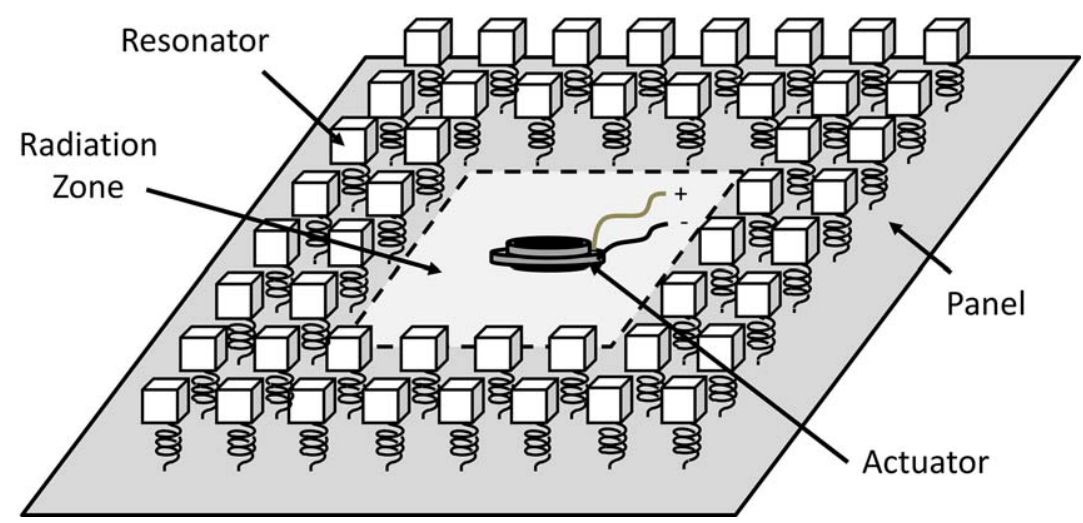

Fig. 12. Panel with band gap structure formed by resonators spaced around a radiation zone with one actuator in the center.

robust by designing filters based on empirical measurements of the panel's vibration profile. Li et al. [97] used vibration contrast control to localize vibrations to a specific region of the panel. An optimization procedure is used to determine a filter for each actuator that maximizes the vibrational energy in one region and minimizes the vibrational energy in all other regions. A similar technique using acoustic contrast was developed by Kournoutos and Cheer [98] for controlling the directivity of panel loudspeakers in which the contrast in the mean-squared pressure in two zones is maximized. Jung et al. [99] was able to eliminate the effects of modal resonances in small-frequency bandwidths using a band gap (BG) structure. The BG structure consists of a periodic arrangement of small resonators distributed on the panel that exponentially reduces the amplitude of bending waves at frequencies within the band prescribed by the structure. This eliminates the presence of bending modes at frequencies within the BG. An example of this is shown in Fig. 12. An experiment that the acoustic resonances displayed by an aluminum panel in the target frequency range of $308-471 \mathrm{~Hz}$ are eliminated by employing a BG structure whose resonators are placed at $25-\mathrm{mm}$ intervals around a $100 \mathrm{~mm} \times 100 \mathrm{~mm}$ radiation zone. With the addition of the BG structure, the variations in sound pressure level radiated from the plate at frequencies within the band were reduced from approximately $40 \mathrm{~dB}$ to approximately $10 \mathrm{~dB}$.

The systems described above for localizing vibrations in the low MO region may be combined with a single actuator positioned at the intended source location via a crossover network to render full-band sound images. A similar method was described for object-based audio rendering on displays, in which different frequency bands are reproduced using different vibration rendering methods [100]. This may have a number of intriguing applications such as pairing sound sources with their corresponding images on the display screen.

There have yet to be significant perceptual studies on the sound quality of localized audio sources rendered by these methods. One study [101] compared the sound fields produced by the methods of [94], a time reversal method
[102], and an inverse filtering method [103, 104]. However, the results are specific to the case of a ribbed plate.

\section{CONCLUSION}

Although flat-panel loudspeakers were invented only a few years after the conventional moving-coil loudspeaker, they have seen relatively little integration into commercial products. Over the years, numerous methods have been developed to tune the frequency response and directivity of flat-panel loudspeakers. The effectiveness of each method is determined by the modal structure of the panel (specifically, the number of modes simultaneously excited at each particular frequency). Some methods, such as adding mechanical damping, are effective for improving the radiation characteristics of panels in the high MO region, while other methods, such as the use of actuator arrays, are more effective in regions of low MO.

Ultimately, flat-panel loudspeakers can produce a sound quality competitive with conventional speakers if they incorporate a combination of such design methods. A crossover network can be used to separate the audio signal into bands so that each band is reproduced using a method that is most effective for that specific frequency range. Integrating these advances in flat-panel loudspeaker technology with modern displays gives one the potential to completely change the user experience in devices such as laptops, smartphones, tablets, and televisions. One can easily imagine displays serving as multimodal interfaces through which virtual environments are created by combining spatial audio and haptic feedback with visual displays.

\section{REFERENCES}

[1] S. E. Olive, "A Multiple Regression Model for Predicting Loudspeaker Preference Using Objective Measurements: Part I - Listening Test Results," presented at the 116th Convention of the Audio Engineering Society (2004 May), convention paper 6113.

[2] S. E. Olive, "A Multiple Regression Model for Predicting Loudspeaker Preference Using Objective Measure- 
ments: Part II - Development of the Model," presented at the 117th Convention of the Audio Engineering Society (2004 Oct), convention paper 6190.

[3] E. W. Kellogg, "Production of Sound," US Patent 1,983,377 (1934 Dec.).

[4] N. Thiele, "Loudspeakers in Vented Boxes: Part 1," J. Audio Eng. Soc., vol. 19, no. 5 , pp. 382-392 (1971).

[5] H. W. Joy, "Diaphragm," US Patent 1,611,454 (1926 Dec.).

[6] "A Note on NXT Distributed Mode Loudspeakers," The Audio Critic, published August 9, 2006, http://biline.ca/audio_critic/audio_critic_web2.htm.

[7] K. Li, A Critical Review of Bending Wave Loudspeaker Technology and Implementation, Master's thesis, Chalmers University of Technology (2010).

[8] S. Lee, Y. Choi, and S. Ham, "Display Apparatus," US Patent 2019/0310684A1 (2019 Oct.).

[9] T.-H. Kim and G.-C. Park, "Display Device," US Patent 2019/0163234A1 (2019 May).

[10] S. Lee, K. Park, K. Jang, and C. Oh, “16-3: Study on Enhancement of the Sound Quality by Improvement of Panel Vibration in OLED TV," in Proceedings of the Society for Information Display International Symposium Digest of Technical Papers, vol. 49, no. 1, pp. 185-187 (2018 May), https://doi.org/10.1002/sdtp.12515.

[11] H. Park, S. Park, and M.-J. Bae, "A Study on the Characteristics of Electroencephalography (EEG) by Listening Location of OLED Flat TV Speaker," in Proceedings of the International Conference on Computational Science/Intelligence \& Applied Informatics, pp. 83-92 (2018), https://doi.org/10.1007/978-3-319-96806-3_7.

[12] Y. Choi, K. Park, S. Lee, and K. Kim, "Panel Vibration Type Display Device for Generating Sound," US Patent 2017/028246A1 (2017 Sep.).

[13] Y. Choi, C. Oh, K. Park, and S. Lee, "Organic Light Emitting Display Device Including A Sound Generating Apparatus," US Patent 9,818,805B2 (2017 Nov.).

[14] L. Cremer, M. Heckl, and B. Petersson, StructureBorne Sound: Structural Vibrations and Sound Radiation at Audio Frequencies (Springer, Berlin, Germany, 2005).

[15] F. Fahy and P. Gardonio, Sound and Structural Vibration: Radiation, Transmission and Response, 2nd ed. (Academic Press, Oxford, United Kingdom, 2007), https://doi.org/10.1016/b978-0-12-373633-8.x5000-5.

[16] Billionsound (website), http://www.billionsound. com/.

[17] B. Wang, C. R. Fuller, and E. K. Dimitriadis, "Active Control of Noise Transmission Through Rectangular Plates Using Multiple Piezoelectric or Point Force Actuators," J. Acoust. Soc. Am., vol. 90, no. 5, pp. 2820-2830 (1991), https://doi.org/10.1121/1.401879.

[18] C. Fuller, C. Hansen, and S. Snyder, "Active Control of Sound Radiation From a Vibrating Rectangular Panel by Sound Sources and Vibration Inputs: An Experimental Comparison," J. Sound Vib., vol. 145, no. 2, pp. 195-215 (1991 Mar.), https://doi.org/ 10.1016/0022-460X(91)90587-A.

[19] E. K. Dimitriadis, C. R. Fuller, and C. A. Rogers, "Piezoelectric Actuators for Distributed Vibration Excita- tion of Thin Plates," J. Vib. Acoust., vol. 113, no. 1, pp. 100-107 (1991 Jan.), https://doi.org/10.1115/1.2930143.

[20] R. L. Clark and C. R. Fuller, "Experiments on Active Control of Structurally Radiated Sound Using Multiple Piezoceramic Actuators," J. Acoust. Soc. Am., vol. 91, no. 6, pp. 3313-3320 (1992), https://doi.org/10.1121/1.402821.

[21] R. L. Clark, M. R. Flemming, and C. R. Fuller, "Piezoelectric Actuators for Distributed Vibration Excitation of Thin Plates: A Comparison Between Theory Experiment," J. Vib. Acoust., vol. 115, no. 3, pp. 332-339 (1993 Jul.), http://dx.doi.org/10.1115/1.2930353.

[22] M. C. Heilemann, Spatial Audio Rendering with Flat-Panel Loudspeakers, Ph.D. thesis, University of Rochester (2018).

[23] C. Fuller, S. Elliott, and P. Nelson, Active Control of Vibration (Academic Press, London, United Kingdom, 1996), https://doi.org/10.1016/b978-0-12-269440-0. x5000-6.

[24] D. A. Anderson, M. C. Heilemann, and M. F. Bocko, "Flat-Panel Loudspeaker Simulation Model with Electromagnetic Inertial Exciters and Enclosures," J. Audio Eng. Soc., vol. 65, no. 9, pp. $722-732$ (2017 Sep.), https://doi.org/10.17743/jaes.2017.0027.

[25] E. G. Williams, Fourier Acoustics (Academic Press, London, United Kingdom, 1999), https://doi.org/10. 1016/B978-0-12-753960-7.X5000-1.

[26] A. Putra and D. Thompson, "Sound Radiation From Rectangular Baffled and Unbaffled Plates," Applied Acoustics, vol. 71, no. 12, pp. 1113-1125 (2010 Dec.), https://doi.org/10.1016/j.apacoust.2010.06.009.

[27] J. A. S. Angus, "Distributed Mode Loudspeaker Polar Patterns," presented at the 107th Convention of the Audio Engineering Society (1999 Sep.), convention paper 5065 .

[28] G. Bank and N. Harris, "The Distributed Mode Loudspeaker-Theory and Practice," presented at the AES 13th UK Conference: Microphones \& Loudspeakers (1998 Mar.), conference paper MAL-18.

[29] J. Panzer and N. Harris, "Distributed Mode Loudspeaker Simulation Model," presented at the 104th Convention of the Audio Engineering Society (1998 May), convention paper 4739 .

[30] H. Azima and P. Mapp, "Diffuse Field DistributedMode Radiators and their Associated Early Reflections," presented at the 104th Convention of the Audio Engineering Society (1998 May), convention paper 4759.

[31] G. Bank, "The Intrinsic Scalability of the Distributed Mode Loudspeaker (DML)," presented at the 104th Convention of the Audio Engineering Society (1998 May), convention paper 4742 .

[32] V. P. Gontcharov and N. P. R. Hill, "Diffusivity Properties of Distributed Mode Loudspeakers," presented at the 108th Convention of the Audio Engineering Society (2000 Feb.), convention paper 5095.

[33] N. Harris, "Modelling Acoustic Room Interaction for Pistonic and Distributed-Mode Loudspeakers in the Correlation Domain," presented at the 117th Convention of the Audio Engineering Society (2004 Oct.), convention paper 6180 . 
[34] E. Prokofieva, K. Horoshenkov, and N. Harris, "Intensity Measurements of the Acoustic Emission From a DML Panel," presented at the 112th Convention of the Audio Engineering Society (2002 Apr.), convention paper 5609.

[35] D. Anderson and M. F. Bocko, "A Model for the Impulse Response of Distributed-Mode Loudspeakers and Multi-Actuator Panels," presented at the 139th Convention of the Audio Engineering Society (2015 Oct.), convention paper 9409.

[36] L. E. Kinsler, A. R. Frey, A. V. Coppens, and J. V. Sanders, Fundamentals of Acoustics, 4th ed. (John Wiley and Sons, Inc., New York, New York, 1999).

[37] D. A. Anderson, M. C. Heilemann, and M. F. Bocko, "Measures of Vibrational Localization on Point-Driven Flat-Panel Loudspeakers," in Proceedings of Meetings on Acoustics Meetings on Acoustics, vol. 26, no. 1 (2016), https://doi.org/10.1121/2.0000216.

[38] M. R. Schroeder, "Statistical Parameters of the Frequency Response Curves of Large Rooms," J. Audio Eng. Soc., vol. 35, no. 5, pp. 299-306 (1987 May).

[39] G. Rabbiolo, R. Bernhard, and F. Milner, "Definition of a High-Frequency Threshold for Plates and Acoustical Spaces," J. Sound Vib., vol. 277, no. 4-5, pp. 647667 (2004 Nov.), http://dx.doi.org/10.1016/j.jsv.2003.09. 015.

[40] C. E. Lane, "Sound Radiator," US Patent 1,773,910 (1930 Aug.).

[41] C. E. Lane, "Sound Radiator," US Patent 1,722,805 (1929 Jul.).

[42] J. V. Nevin, "Screen Loud Speaker," US Patent 1,778,084 (1930 Oct.).

[43] J. C. Kroesen, "Picture Screen," US Patent 1,817,630 (1931 Aug.).

[44] H. Sotome, "Loudspeaker," US Patent 3,483,946 (1969 Dec.).

[45] Y. Mochida, "Flat-Plate Type Loudspeaker With Frame Mounted Drivers," US Patent 3,509,290 (1970 Apr.).

[46] J. Bertagni, "Flat Diaphragm for Sound Transducers and Method for Manufacturing It," US Patent 3,596,733 (1971 Aug.).

[47] J. Bertagni, "Flat Diaphragm for Sound Transducers," US Patent 3,722,617 (1973 Mar.).

[48] J. Bertagni, "Flat Loudspeaker with Enhanced Low Frequency," US Patent 3,767,005 (1973 Oct.).

[49] J. Bertagni, "Planar Diaphragm," US Patent 4,003,449 (1977 Jan.).

[50] J. Bertagni, "Mounting of a Substantially Planar Diaphragm Defining a Sound Transducer," US Patent 4,257,325 (1981 Mar.).

[51] K. H. Heron, "Panel-Form Loudspeaker," US Patent 6,058,196 (2000 May).

[52] H. Azima, M. Colloms, and N. Harris, "Panel-Form Loudspeakers," US Patent 6,031,926 (2000 Feb.).

[53] H. Azima, "Acoustic Device," US Patent 6,694,038 (2004 Feb.).

[54] N. Harris and G. Bank, "A Balanced Mode Radiator (BMR)," presented at the 119th Convention of the Audio Engineering Society (2005 Oct.), convention paper 6595.
[55] D. Beer, B. Schlenker, S. Brix, and T. Sporer, "Loudspeaker," US Patent 7,391,879 (2008 Jun.).

[56] A. Hill, "Acoustic Transducer," US Patent 4,928,312 (1990 May).

[57] C. Lock, J. Corey, G. Jones, and K. Waterman, "Loudspeakers," US Patent 6,411,723 (2002 Jun.).

[58] X. Shen, Y. Shen, and Y. Dong, "Modal Optimization of Distributed Mode Loudspeaker," presented at the 118th Convention of the Audio Engineering Society (2005 May), convention paper 6522.

[59] S. Zhang, Y. Shen, X. Shen, and J. Zhou, "Modal Optimization of Distributed-Mode Loudspeaker Using Attached Masses," J. Audio Eng. Soc., vol. 54, no. 4, pp. 295-305 (2006 Apr.).

[60] M. J. Hawksford, "MATLAB Program for Loudspeaker Equalization and Crossover Design," J. Audio Eng. Soc., vol. 47, no. 9, pp. 706-719 (1999).

[61] M. M. Boone, "Multi-Actuator Panels (MAPs) as Loudspeaker Arrays for Wave Field Synthesis," J. Audio Eng. Soc., vol. 52, no. 7/8, pp. 712-723 (2004 Jul.).

[62] B. Zenker, S. Rawoof, S. Merchel, and M. Altinsoy, "Low Deviation and High Sensitivity-Optimized Exciter Positioning for Flat Panel Loudspeakers by Considering Averaged Sound Pressure Equalization," presented at the 147th Convention of the Audio Engineering Society (2019 Oct.), convention paper 10246, https://doi.org/10.17743/ aesconv.2019.978-1-942220-31-2.

[63] S. Zhang, Y. Shen, and X. Shen, "Positions Effect of Multi Exciters and the Optimization on Sound Pressure Responses of Distributed Mode Loudspeaker," presented at the 120th Convention of the Audio Engineering Society (2006 May), convention paper 6841.

[64] D. Anderson and M. F. Bocko, "Modal Crossover Networks for Flat-Panel Loudspeakers," J. Audio Eng. Soc., vol. 64, no. 4, pp. 229-240 (2016 Apr.), http://dx.doi.org/ 10.17743/jaes.2016.0005.

[65] D. Anderson, M. C. Heilemann, and M. F. Bocko, "Optimized Driver Placement for Array-Driven Flat-Panel Loudspeakers," Arch. Acoust., vol. 42, no. 1, pp. 93-104 (2017), https://doi.org/10.1515/aoa-2017-0010.

[66] D. Anderson, M. Heilemann, and M. F. Bocko, "Impulse and Radiation Field Measurements for Single Exciter Versus Exciter Array Flat-Panel Loudspeakers," presented at the 143rd Convention of the Audio Engineering Society (2017 Oct.), eBrief 379, https://doi.org/10.17743/ aesconv.2017.978-1-942220-18-3.

[67] F. H. Brittain, "The Appraisement of Loudspeakers, Part I,” GEC J., vol. 7, pp. 266-277 (1936).

[68] F. H. Brittain, "The Appraisement of Loudspeakers, Part II," GEC J., vol. 8, pp. 121-130 (1937).

[69] F. H. Brittain, "Loudspeakers: Relations Between Subjective and Objective Tests," J. Brit. Inst. Radio Eng., vol. 13, no. 2, pp. 105-109 (1953 Feb.), https://doi.org/10.1049/jbire.1953.0010.

[70] F. E. Toole, "Loudspeaker Measurements and Their Relationship to Listener Preferences: Part 1," J. Audio Eng. Soc., vol. 34, no. 4, pp. 227-235 (1986 Apr.).

[71] F. E. Toole, "Subjective Measurements of Loudspeaker Sound Quality," presented at the 72nd Convention 
of the Audio Engineering Society (1982), convention paper 1900.

[72] F. E. Toole, "Subjective Measurements of Loudspeaker Sound Quality and Listener Performance," J. Audio Eng. Soc., vol. 33, no. 1/2, pp. 2-32 (1985 Feb.).

[73] F. E. Toole, "Loudspeaker Measurements and Their Relationship to Listener Preferences: Part 2," J. Audio Eng. Soc., vol. 34, no. 5, pp. 323-348 (1986 May).

[74] S. Flanagan and V. Taylor, "Investigation Into the Relationship Between Subjective Loudness and Auditory Distance Perception," presented at the 107th Convention of the Audio Engineering Society (1999 Sep.), convention paper 5049.

[75] S. Flanagan and N. Harris, "Loudness: A Study of the Subjective Difference between DML and Conventional Loudspeaker," presented at the 106th Convention of the Audio Engineering Society (1999 May), convention paper 4872.

[76] N. Harris, S. Flanagan, and M. J. Hawksford, "Stereophonic Localization in the Presence of Boundary Reflections, Comparing Specular and Diffuse Acoustic Radiators," presented at the 104th Convention of the Audio Engineering Society (1998 May), convention paper 4684 .

[77] N. J. Harris, The Acoustics and Psychoacoustics of the Distributed-Mode Loudspeaker (DML), Ph.D. thesis, University of Essex (2001).

[78] M. Heilemann, D. Anderson, S. Roessner, and M. F. Bocko, "Quantifying Listener Preference of Flat-Panel Loudspeakers," presented at the 145th Convention of the Audio Engineering Society (2018 Oct.), convention paper 10087, https:// doi.org/10.17743/aesconv.2018.978-1-942220-25-1.

[79] S. Roessner, M. Heilemann, and M. F. Bocko, "Evaluating Listener Preference of Flat-Panel Loudspeakers," presented at the 147th Convention of the Audio Engineering Society (2019 Oct.), convention paper 10230, https://doi.org/10.17743/aesconv.2019.978-1-942220-31-2.

[80] A. J. Berkhout, D. de Vries, and P. Vogel, "Acoustic Control by Wave Field Synthesis," J. Acoust. Soc. Am., vol. 93, no. 5, pp. 2764-2778 (1993), https://doi.org/10.1121/1.405852.

[81] W. van Rooijen, Distributed Mode Loudspeakers for Wave Field Synthesis, Master's thesis, Delft University of Technology (2001).

[82] E. Corteel, U. Horbach, and R. Pellegrini, "Multichannel Inverse Filtering of Multiexciter Distributed Mode Loudspeakers for Wave Field Synthesis," presented at the 112th Convention of the Audio Engineering Society (2002 Apr.), convention paper 5611.

[83] B. Pueo, J. J. López, G. Ramos, and J. Escolano, "Efficient Equalization of Multi-Exciter Distributed Mode Loudspeakers," Appl. Acoust., vol. 70, no. 5, pp. 737-746 (2009 May), https://doi.org/10.1016/ j.apacoust.2008.09.005.

[84] B. Pueo, J. Escolano, J. J. Lopez, and G. Ramos, "A Note on the Filtering Equalization in Large Multiactuator Panels," presented at the 2009 17th European Signal Processing Conference, pp. 799-803 (2009 Aug).
[85] J.-H. Ho and A. P. Berkhoff, "Flat Acoustic Sources With Frequency Response Correction Based on Feedback and Feed-Forward Distributed Control," $J$. Acoust. Soc. Am., vol. 137, no. 4, pp. 2080-2088 (2015), https://doi.org/10.1121/1.4914997.

[86] B. Pueo, G. Ramos, and J. J. Lopez, "Strategies for Bass Enhancement in Multiactuator Panels for Wave Field Synthesis," Appl. Acoust., vol. 71, no. 8, pp. 722-730(2010 Aug.), https://doi.org/10.1016/j.apacoust.2010.03.003.

[87] M. Kuster, D. De Vries, X. Beer, and S. Brix, "Structural and Acoustic Analysis of Multiactuator Panels," J. Audio Eng. Soc., vol. 54, no. 11, pp. 1065-1076 (2006).

[88] J. van Dorp Schuitman and D. de Vries, "Wave Field Synthesis Using Multi-Actuator Panel: Further Steps to Optimal Performance," presented at the AES 28th International Conference: The Future of Audio TechnologySurround and Beyond (2006 Jun.), conference paper 4-3.

[89] M. Rébillat, E. Corteel, B. F. Katz, and X. Boutillon, "From Vibration to Perception: Using Large Multi-Actuator Panels (LaMAPs) to Create Coherent Audio-Visual Environments," presented at the S. F. d'Acoustique, Acoustics 2012 Conference (2012 Apr.).

[90] O. Jeon, H. Jeon, and W. Semyung, "Optimal Design of Exciter Array to Minimize Bending Mode Effect of Multi-Actuator Panels in Beamforming," in Proceedings of the INTER-NOISE and NOISE-CON Congress and Conference, vol. 259, pp. 299-303 (2019).

[91] B. Pueo, J. J. López, J. Escolano, and L. Hörchens, "Multiactuator Panels for Wave Field Synthesis: Evolution and Present Developments," J. Audio Eng. Soc., vol. 58, no. 12, pp. 1045-1063 (2010 Dec.).

[92] J.-H. Woo and J.-G. Ih, "Vibration Rendering on a Thin Plate With Actuator Array at the Periphery," J. Sound Vib., vol. 349, pp. 150-162 (2015), https://doi.org/10.1016/ j.jsv.2015.03.031.

[93] L. Meirovitch and J. Bennighof, "Modal Control of Traveling Waves in Flexible Structures," J. Sound Vib., vol. 111, no. 1, pp. 131-144 (1986 Nov.), http://dx.doi.org/ 10.1016/S0022-460X(86)81428-6.

[94] M. C. Heilemann, D. Anderson, and M. F. Bocko, "Sound-Source Localization On Flat-Panel Loudspeakers," J. Audio Eng. Soc., vol. 65, no. 3, pp. 168-177 (2017 Mar.), https://doi.org/10.17743/jaes.2016.0066.

[95] M. C. Heilemann, D. Anderson, and M. F. Bocko, "Equalization of Localized Sources on Flat-Panel Audio Displays," presented at the 143rd Convention of the Audio Engineering Society (2017 Oct.), convention paper 9871, https://doi.org/10.17743/aesconv.2017.978-1-942220-18-3.

[96] M. C. Heilemann, D. Anderson, and M. F. Bocko, "Source Rendering on Dynamic Audio Displays," in Proceedings of the IEEE Workshop on Applications of Signal Processing to Audio and Acoustics (WASPAA), pp. 334-338 (2017 Oct.), https://doi.org/10.1109/ waspaa.2017.8170050.

[97] Z. Li, P. Luo, C. Zheng, and X. Li, "Vibrational Contrast Control for Local Sound Source Rendering on Flat Panel Loudspeakers," presented at the 
145th Convention of the Audio Engineering Society (2018 Oct.), convention paper 10086, https://doi.org/10.17743/ aesconv.2018.978-1-942220-25-1.

[98] N. Kournoutos and J. Cheer, "A System for Controlling the Directivity of Sound Radiated From a Structure," J. Acoust. Soc. Am., vol. 147, no. 1, pp. 231-241 (2020), https://doi.org/10.1121/10.0000589.

[99] J. Jung, C.-H. Jeong, and J. S. Jensen, "Spectrally Smooth and Spatially Uniform Sound Radiation From a Thin Plate Structure Using Band Gaps," $J$. Sound Vib., vol. 471, p. 115187 (2020), https://doi.org/ 10.1016/j.jsv.2020.115187.

[100] M. C. Heilemann, D. A. Anderson, and M. F. Bocko, "Near-Field Object-Based Audio Rendering on Flat-Panel Displays," J. Audio Eng. Soc., vol. 67, no. 7/8, pp. 531-539 (2019 Jul.), https://doi.org/10.17743/ jaes.2019.0034.
[101] N. Benbara, M. Rebillat, and M. Nazih, "Bending Waves Focusing in Arbitrary Shaped Plate-Like Structures: Application to Spatial Audio," in Proceedings of the 23rd International Congress on Acoustics (2019).

[102] M. Fink, "Time Reversal of Ultrasonic Fields. I. Basic Principles," IEEE Trans. Ultrason. Ferroelectr. Freq. Control, vol. 39, no. 5, pp. 555-566 (1992 Sep.), https://doi.org/10.1109/58.156174.

[103] Y. Kahana, P. A. Nelson, O. Kirkeby, and H. Hamada, "A Multiple Microphone Recording Technique for the Generation of Virtual Acoustic Images," $J$. Acoust. Soc. Am., vol. 105, no. 3, pp. 1503-1516 (1999), https://doi.org/10.1121/1.426690.

[104] M. Tanter, J.-L. Thomas, and M. Fink, "Time Reversal and the Inverse Filter," J. Acoust. Soc. Am., vol. 108, no. 1, pp. 223-234 (2000), https://doi.org/10.1121/ 1.429459 .

THE AUTHORS

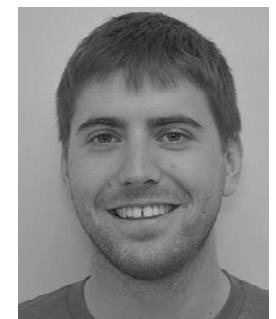

Michael C. Heilemann

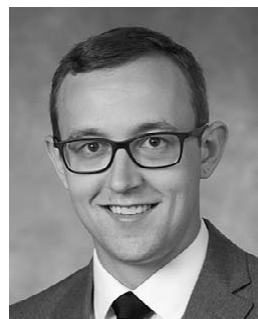

David A. Anderson

Michael C. Heilemann received his M.S. and Ph.D. in Electrical Engineering from the University of Rochester in 2015 and 2018, respectively. In 2017, he was named the Harman Scholar by the Audio Engineering Society Educational Foundation. After completing his doctoral studies, he joined the faculty at the University of Rochester in the Department of Electrical and Computer Engineering. His research interests are in the areas of electroacoustics and spatial audio. Professor Heilemann advises the capstone projects for students majoring in Audio and Music Engineering and teaches courses on signal processing and acoustics.

David A. Anderson is an Assistant Professor in the Electrical Engineering department at the University of Colorado Denver. He completed his Ph.D. at the University of Rochester in 2017 with research on excitation systems for flat-panel loudspeakers. From 2018 to 2020, he was an Assistant Professor at the University of Pittsburgh. He teaches courses on audio, electronics, circuits, and engineering design. His research interests include loudspeaker system design, haptics, and active acoustic sensing.

$$
\text { - }
$$

Stephen Roessner is a Grammy Award-winning audio engineer and Senior Lecturer at the University of Rochester.

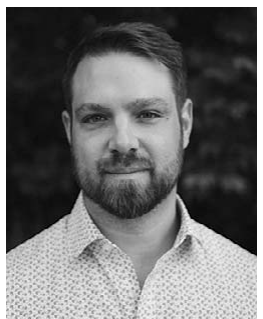

Stephen Roessner

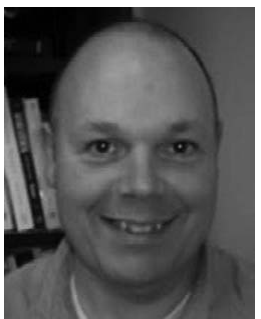

Mark F. Bocko
His research interests include acoustics, data mining of musical preferences, and audio education practices. He currently performs with his band Fuzzrod and as a solo musician under the name Small Signals.

Mark F. Bocko earned his Ph.D. in Physics from the University of Rochester in 1984. After a brief postdoctoral appointment, he joined the university's Electrical and Computer Engineering Department in 1985. His research has spanned multiple areas, with its current focus being on audio and acoustic signal processing. He is also the Director of the Center for Emerging and Innovative Sciences (CEIS), a New York State Office of Science, Technology and Academic Research (NYSTAR) supported Center for Advanced Technology at the University of Rochester. Professor Bocko has taught courses on solid state devices, microwaves, circuits and systems, audio signal processing, and acoustics. He was won five teaching awards at the University of Rochester and was named the Mercer Brugler Distinguished Teaching Professor at the university from 2008 to 2011. He was named Distinguished Professor of Electrical and Computer Engineering in 2013 and served as Chair of the Electrical and Computer Engineering Department from 2004 to 2010 and again from 2012 to 2020. 